\title{
Mutation detection in the aspartoacylase gene in 17 patients with Canavan disease: four new mutations in the non-Jewish population
}

\author{
Erik A Sistermans ${ }^{1}$, René FM de $\mathrm{Coo}^{1,4}$, Hetty $M$ van Beerendonk ${ }^{1}$, Bwee Tien Poll-The ${ }^{2}$, \\ Wim J Kleijer ${ }^{3}$ and Bernard A van Oost ${ }^{1,5}$
}

\begin{abstract}
${ }^{1}$ D epartment of Human Genetics, University Hospital, Nijmegen; ${ }^{2}$ Department of M etabolic Disorders, W ilhelmina Children's Hospital, Utrecht Medical Centre; ${ }^{3}$ Department of Clinical Genetics, University Hospital, Rotterdam, The Netherlands
\end{abstract}

Canavan disease is a severe progressive autosomal recessive disorder, which is characterised by spongy degeneration of the brain. The disease is caused by mutations in the aspartoacylase gene. Two different mutations were reported on $\mathbf{9 8 \%}$ of the alleles of Ashkenazi Jewish patients, in which population the disease is highly prevalent. In non-Jewish patients of European origin, one mutation (914C $>A$ ) is found in $50 \%$ of the alleles, the other alleles representing all kinds of different mutations. We here describe the results of the mutation analysis in 17 European, non-Jewish patients. Ten different mutations were found, of which four had not been described before (H21P, A57T, R168H, P181T). A deletion of exon 4, which until now had only been described once, was revealed in all five alleles of Turkish origin tested, indicating that this is a founder effect in the Turkish population. European Journal of Human Genetics (2000) 8, 557-560.

Keywords: Canavan disease; aspartoacylase; mutation detection; $\mathrm{N}$-acetylaspartic acid; prenatal diagnosis

\section{Introduction}

Canavan disease (OMIM 271900) is an autosomal recessive lethal neurodegenerative disorder characterised by spongy degeneration of the brain. The most common clinical features are hypotonia, megal encephaly, severe mental retardation, optic atrophy and early death. The disease is caused by a deficiency of aspartoacylase (ASPA), an enzyme that catalyses the hydrolysis of $\mathrm{N}$-acetylaspartic acid (NAA) into aspartate and acetate. ${ }^{1}$ The cloning of the ASPA gene (Genbank S67156) allowed mutation analysis for Canavan disease. $^{2}$ To date, more than 20 different mutations in the ASPA gene have been reported, including two Ashkenazi Jewish (98\% of alleles) and one Pan-European (50\%) founder mutation. In the non-Jewish population the other alleles

Correspondence: Erik A Sistermans, PO Box 9101, 6500 HB Nijmegen, The Netherlands. Tel: +31243613 799; Fax: +312436 16658; E-mail: E.Sistermans@antrg.azn.nl

${ }^{4}$ Current address: Department of Child Neurology, University Hospital, Rotterdam, The Netherlands

${ }^{5}$ Current address: Department of Clinical Sciences of Companion Animals, Utrecht, The Netherlands

Received 7 September 1999; revised 18 January 2000; accepted

21 January 2000 consist of different mutations spread throughout the gene. $^{3-5}$

Canavan disease is diagnosed by measuring the elevated levels of NAA in urine or cerebro-spinal fluid, or enzymatically in cultured skin fibroblasts. ${ }^{6}$ Both methods, however, have drawbacks as there is a wide variation in the amount of NAA excreted and because the enzyme assay is complex and very sensitive to assay conditions. Furthermore, prenatal diagnosis on the basis of an enzymatic assay on aminocytes and chorion villi has been reported unreliable. ${ }^{7}$ Therefore, preference should be given to molecular techniques for performing reliable prenatal diagnosis.

\section{Materials and methods}

Samples from 17 families reported to be from non-Jewish European descent were analysed. All patients were diagnosed by the demonstration of increased level s of NAA in urine. All patients were sporadic cases, except for the monozygotic twins of family 1 , and the two brothers of family 16 . In the latter family the patients had died and the parents were analysed in order to allow carrier analysis for their daughter. Genomic DNA was isolated by a simple salt-precipitation 
method. $^{8}$ Total RNA was isolated using RNAzol ${ }^{\text {TM }}$ (CAMPRO Scientific, Veenendaal, The Netherlands). cDNA was generated with Superscript ${ }^{T M}$ II Rnase $\mathrm{H}$ reverse transcriptase (Life Technologies, Breda, The Netherlands) using random sequence hexadeoxynucleotides (Promega, Leiden, The Netherlands).

The $914 \mathrm{C}>\mathrm{A}$ mutation was analysed as described ${ }^{9}$ although the forward primer was replaced by $632 \mathrm{~F}$ (5'TTAACTCTTGATGGGAAGACGATC3') to allow the identification of the mutation at the DNA level. The same fragment was used for the analysis of the 854A >C mutation, which causes gain of both an Eagl and a Notl site. The 693C/T >A mutation, which results in gain of an Msel site, was assayed at the DNA level by amplifying exon 5 with the primers $4 \mathrm{~F}$ and 3R (see below).

The primer sets used for preparing the four overlapping fragments used for single-strand conformation polymorphism (SSCP) analysis and sequencing are:

\section{IF 5'CTTTGATCTCTCTTCTGAATTGCA3';}

1R 5'CAGGTCACAGTCAATATATCTGGTAC3'; 2F 5'CAGGGCTGGAGGTAAAACCA3';

2R 5'GCATATITGAGGGAAGGATG3';

\section{F 5'CATTACATTAAGACTTCTCTGGCT3';}

3R 5'CCTGCAGATTAGGATGG3';

\section{F 5'AAGAATTTCCTCCCTGCGCC3';}

\section{R 5'GTAAGACACCGTGTAAGATGTAAGCT3'.}

SSCP analysis was performed on a GenePhor electrophoresis unit using the accompanying GeneGel Excel 12.5/ 24 kit. Fixation and staining (5') was performed in a Hoefer ${ }^{\mathrm{TM}}$ automated gel stainer using the PlusOne DNA silver staining kit (Amersham Pharmacia Biotech, Roosendaal, The Netherlands).

Sequencing of the fragments was performed using a dye terminator sequencing kit (PE-ABI, Nieuwerkerk a/d IJssel, The Netherlands). Samples were analysed on an ABI 373 automatic sequencer (PE-ABI).

The $62 \mathrm{~A}>\mathrm{C}$ mutation results in gain of a BstNI site and loss of an Ncol site. The 169G >A mutation was analysed using primer $1 \mathrm{~F}$ and primer 1647R (5'CAATATATCTGGTACACTTCTTCACAG3') which introduces an Alul site which is disrupted by the mutation. PCR with primers $\mathrm{HASP}^{4} \mathrm{~B}^{4}$ and 1425F (5'CCTCAAATATGCGACCACGC3') creates an Mlul site which is disrupted by the $503 \mathrm{G}>\mathrm{A}$ mutation. Mutation $541 C>A$, results in gain of an Rsal site.
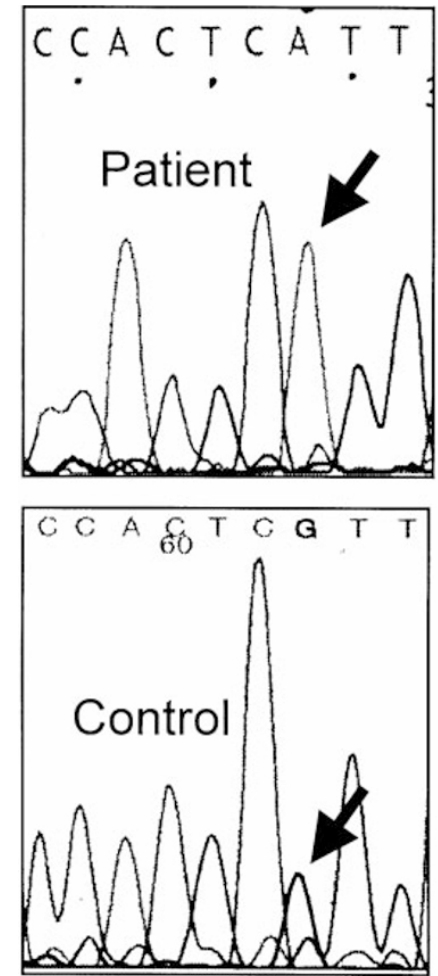
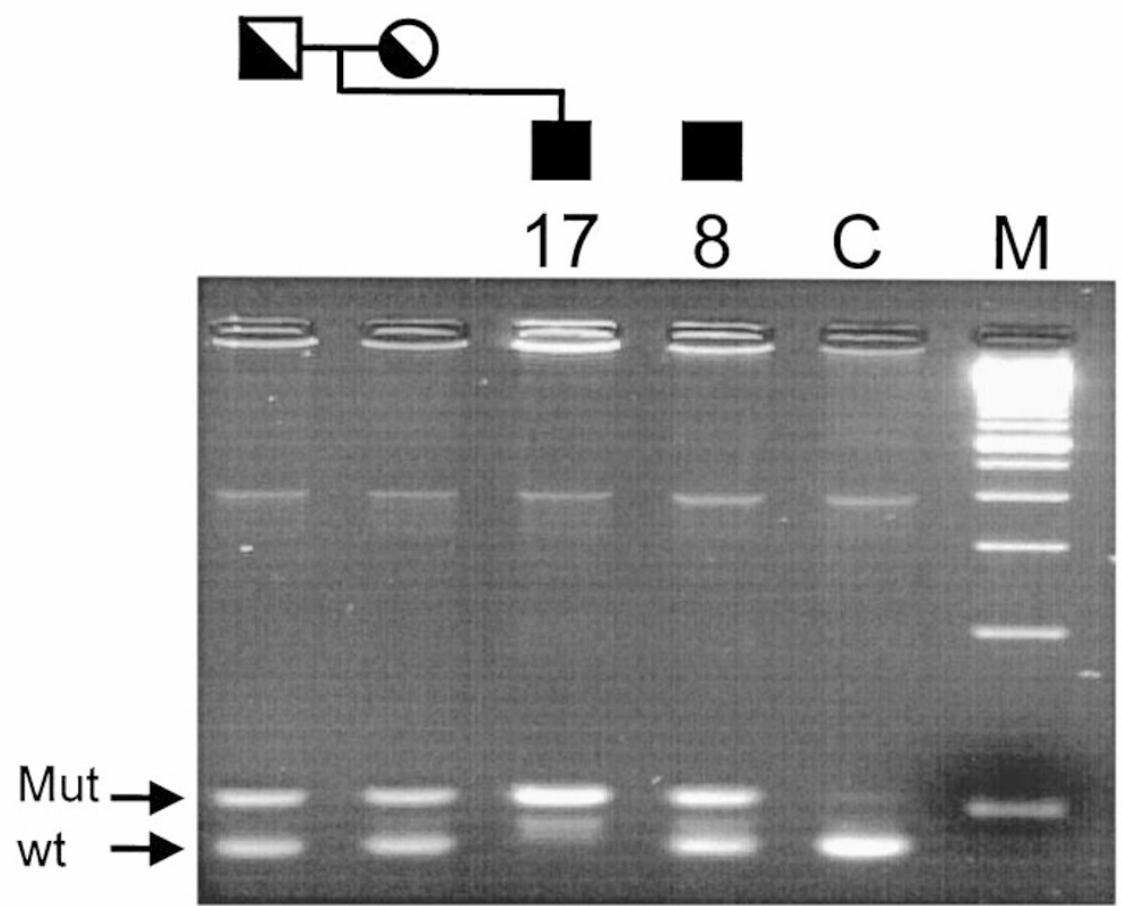

Figure 1 Mutation 503G - >A (R168H). Results of the sequence analysis are shown for patient 17, who is homozygote for this mutation (left panels). Digestion with Mlul revealed that both parents of patient 17 were heterozygous carriers of this mutation, as was patient 8: C; control sample: M; 100bp marker, arrows indicate the wild-type and mutant bands. 


\section{Results}

All patients were first analysed for the common panEuropean $914 \mathrm{C}>\mathrm{A}$ mutation. This mutation was found in 17 of the 34 alleles tested (50\%), seven patients being homozygous and three being heterozygous. Of the common

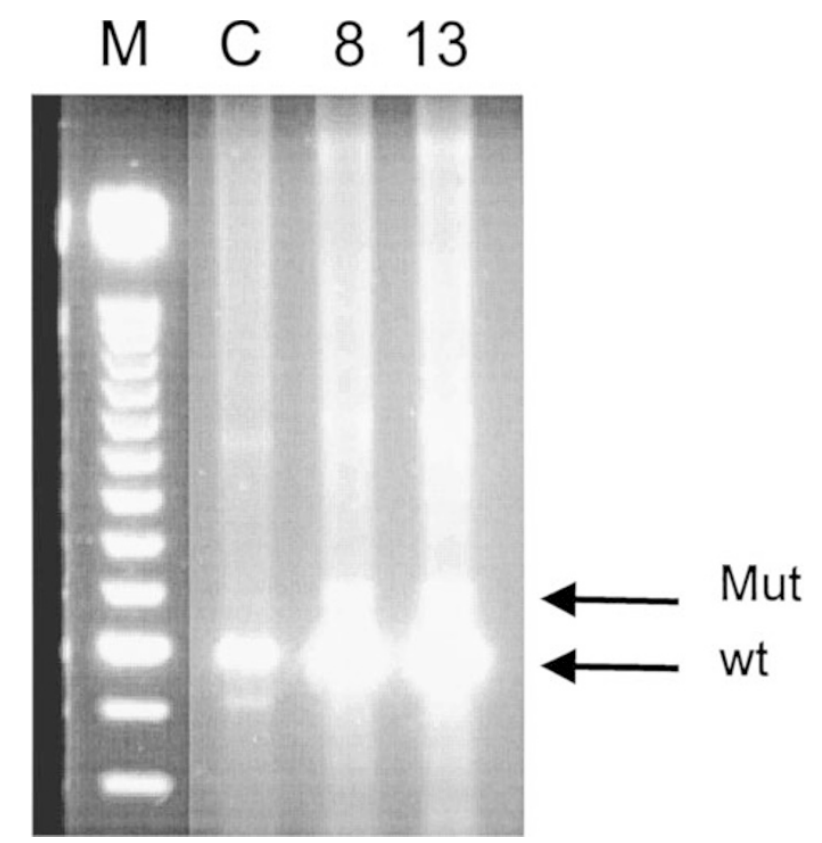

Figure 2 Putative splicing mutation in patients 8 and 13. In these patients a discrepancy was seen between sequencing results on CDNA (homozygous mutations) and DNA (heterozygous mutations). Generation of a 3' cDNA fragment comprising exons 3-6 revealed a faint larger fragment: C; control sample: $\mathrm{M}$; $100 \mathrm{bp}$ marker, arrows indicate the wild-type and mutant bands.
Jewish mutations only the $693 \mathrm{C}>\mathrm{A}$ mutation was found on one allele of patient 10.

DNA samples of the 10 patients that were not homozygous for the $914 \mathrm{C}>\mathrm{A}$ mutation were further analysed. Two patients were pre-screened by SSCP analysis followed by sequence analysis. This approach revealed 169G $>$ A (A57T) and 244-245insA mutations in patient 12 and a $62 \mathrm{~A}>\mathrm{C}$ (H21P) mutation in patient 14.

DNA samples of the remaining eight patients were directly sequenced, revealing four more mutations. A $541 \mathrm{C}>\mathrm{A}$ (P181T) transversion was found in both patients from family 1 . Mutation 503G >A (R168H) was present heterozygous in the English patient and homozygous in a Belgian patient (Figure1), whereas 79G >A (G27R) was found in patient 9. Finally an in-frame del etion of exon 4 was detected on all five alleles of Turkish origin tested.

In two patients $(8,13)$ where only one mutation was found, the mutation was homozygous at the CDNA level but heterozygous at the DNA level. Analysis of the $3^{\prime}$ half of the cDNA of these patients revealed a faint band of increased length, not present in control cDNA (Figure2). It was impossible to sequence the longer fragment, as it could not be purified at sufficiently high levels. Neither could the underlying mutation be identified at the DNA level, as the intronic sequences are unknown. A summary of the results is given in Table1.

\section{Discussion}

The mutational spectrum within the non-Jewish population is more complex than in the Ashkenazi Jewish population. (An overview of all known mutations is available at the Human Gene Mutation Database, http://www.uwcm.ac.uk/ uwcm/mg/hgmd0.html). Although the frequency of the common $914 \mathrm{C}>\mathrm{A}$ mutation (50\%) in the 17 patients was

Table 1 Overview of the results of the mutation analysis in 17 patients with Canavan disease. In those cases where the parents could be tested to prove that the mutations were located on different alleles, the paternal mutation is indicated as mutation 1 , the maternal mutation as mutation 2. Mutations that have not been described before are in italics

\begin{tabular}{|c|c|c|c|c|c|c|}
\hline Patient & Mutation 1 DNA & Mutation 2 DNA & Mutation 1 protein & Mutation 2 protein & Parents tested & Ethnic background \\
\hline 1 & $541 C->A$ & del exon 4 & Prol81Thr & 193del36 & yes & Dutch/Turkish \\
\hline 2 & del exon 4 & del exon 4 & 193del36 & 193del36 & yes & Turkish \\
\hline 3 & $914 C->A$ & $914 C->A$ & Ala305Glu & Ala305Glu & yes & Dutch \\
\hline 4 & $914 C->A$ & $914 C->A$ & Ala305Glu & Ala305Glu & no & Czech \\
\hline 5 & del exon 4 & del exon 4 & 193del36 & 193del36 & no & Turkish \\
\hline 6 & $914 C->A$ & $914 C->A$ & Ala305Glu & Ala305Glu & no & Czech \\
\hline 7 & $914 C->A$ & $914 C->A$ & Ala305Glu & Ala305Glu & no & Czech \\
\hline 9 & $914 C->A$ & $79 \mathrm{G}->A$ & Ala305Glu & Gly27Arg & yes & Dutch \\
\hline 10 & $693 C->A$ & - & Tyr231Ter & - & father & South African \\
\hline 11 & $914 C->A$ & $914 C->A$ & Ala305Glu & Ala305Glu & yes & Dutch \\
\hline 12 & 244-245insA & $169 \mathrm{G}->\mathrm{A}$ & Frameshift & Ala57Thr & no & German \\
\hline 13 & $914 C->A$ & splicing & Ala305Glu & unknown & no & Czech \\
\hline 14 & $62 A->C$ & $914 C->A$ & His21Pro & Ala305Glu & yes & Dutch \\
\hline 15 & $914 C->A$ & $914 C->A$ & Ala305Glu & Ala305Glu & yes & Belgian \\
\hline 16 & $914 C->A$ & $914 C->A$ & Ala305Glu & Ala305Glu & yes & Dutch \\
\hline 17 & $503 G->A$ & $503 G->A$ & Arg168His & Arg168His & yes & Belgian \\
\hline
\end{tabular}


comparable with earlier studies, ${ }^{3-5}$ differences were observed between countries of origin. It was found on $88 \%$ of the eight Czech alleles but was absent in all five alleles of Turkish origin tested. These alleles all carried an in-frame deletion of exon 4 which has been described before in a Turkish patient born of consanguineous patients. ${ }^{3}$ The results presented here assign this deletion as a common mutation in the Turkish population.

Three more recurrent mutations were identified, 79G $>A,{ }^{4}$ 244-245ins $\mathrm{A}^{5}$ and $503 \mathrm{G}>\mathrm{A}$ which has not been reported before, but was found on three independent alleles. These mutations should be screened for when molecular analysis is performed in non-Jewish patients.

Not much is known about the structural properties of aspartoacylase, making it difficult to predict the effect of the new mutations (H21P, A57T, R168H, P181T) on the enzymatic activity. However, all residues involved are highly conserved between the unrelated species human, bovine, and Cynechocystis sp., H21, A57 and P181 even in Prochlorococcus marinus. Furthermore, the mutations were not found in 200 control alleles. Finally, most mutations involve nonconserved amino-acid changes, except for 503G >A. However, this mutation affects the same residue as the $\mathrm{R} 168 \mathrm{C}$ mutation which renders the protein completely inactive. ${ }^{4}$ Of high interest is the 62A $>\mathrm{C}$ mutation as it affects the His 21 residue, which is an invariable residue in the first esterase consensus sequence present in the protein. This residue was predicted to be part of a Ser, His, Glu triad that forms the catalytic domain. ${ }^{3}$ In analogy with the common Jewish $854 \mathrm{~A}>\mathrm{C}$ mutation that involves the Glu residue of this triad, it can be predicted that substitution of the His residue will al so result in inactivation of ASPA.

In conclusion, mutations found in Canavan disease patients not only differ between Jewish and non-Jewish patients, but also within the non-Jewish population. Prior knowledge of the ethnic background of a patient should therefore be obtained before mutation analysis is initiated.

\section{References}

1 Matalon R, Michals K, Sebasta D, Deanching M, Gashkoff P, Casanova J: Aspartoacylase deficiency in $\mathrm{N}$-acetylaspartic aciduria in patients with Canavan disease. Am J Med Genet 1988; 29: 463-471.

2 Kaul R, Gao GP, Balamurugan K, Matalon R: Cloning of the human aspartoacylase CDNA and a common missense mutation in Canavan disease. Nat Genet 1993; 5: 118-123.

3 Shaag A, Anikster Y, Christensen E et al: The molecular basis of Canavan (Aspartoacylase deficiency) disease in European nonJewish patients. Am J Hum Genet 1995; 57: 572-580.

4 Kaul R, Gao GP, Matalon R et al: Identification and expression of eight novel mutations among non-Jewish patients with Canavan disease. Am J Hum Genet 1996; 59: 95-102.

5 Elpeleg ON, Shaag A: The spectrum of mutations of the aspartoacylase gene in Canavan disease in non-Jewish patients. J Inher M etab Dis 1999; 22: 531-534.

6 Matalon R, Kaul R, Michals K: Canavan disease: Biochemical and molecular studies. J Inher Metab Dis 1993; 16: 744-752.

7 Matalon R, Michals K, Gashkoff P, Kaul R: Prenatal diagnosis of Canavan disease. J Inher Metab Dis 1992; 15: 392-394.

8 Millar SA, Dykes DD, Polesky HF: A simple salting out procedure for extracting DNA from human nucleated cells. Nucleic Acids Res 1988; 16: 1215.

9 Kaul R, Gao GP, Aloya M et al: Canavan disease: Mutations among Jewish and Non-Jewish patients. Am J Hum Genet 1994; 55: 34-41. 